\title{
Mortalidad por accidentes laborales en del Departamento del Meta, años 2010-2015
}

\author{
Occupational mortality in the Meta Department, 2010-2015 \\ Mortalidade por acidentes de trabalho no Departamento de Meta, anos 2010-2015 \\ Laura Inés Plata-Casas*
}

\section{Resumen}

Introducción: La mortalidad es considerada un factor de medición del estado de salud poblacional, por tanto, resulta indispensable reconocer su comportamiento epidemiológico. Objetivo: Determinar la epidemiología de los casos de mortalidad por accidente laboral ocurridos en el Meta, durante el periodo comprendido entre 20102015. Materiales y Métodos: Estudio descriptivo y análisis de información del registro único de afiliaciones, procesada en SPSS. Se utilizarán: estadística descriptiva, tasas de mortalidad y análisis de tendencias. Resultados: La tendencia en los casos de mortalidad por accidentes laborales ha disminuido, el $81 \%$ de la variabilidad de los casos de mortalidad, es explicada por su relación con los años. Conclusión: Los proyectos, programas y políticas de salud deben reflejar un seguimiento y monitoreo a los programas de promoción de la salud laboral. Igualmente, se debe evaluar su impacto, así como la realización de ajustes y su priorización.

Palabras Clave: Mortalidad Laboral, Accidentes de Trabajo, Colombia.

\section{Abstract}

Introduction: Mortality is considered a measuring factor of the population health; therefore, it is indispensable to recognize its epidemiological behavior. Objective: Determine the epidemiology of the occupational mortality cases in the Meta Department during the 2010-2015 period. Materials and Methods: Descriptive study and analysis of information from single affiliation records, processed in SPSS. Descriptive statistics, mortality rates and trend analysis will be used. Results: The tendency in occupational mortality has decreased, $81 \%$ of the variability in the occupational mortality cases has been explained by its relation with age. Conclusion: The health projects, programs, and policies should reflect follow-up and monitoring of promotion programs in the occupational health area. Also, its impact must be evaluated, as well as conducting adjustments and prioritization.

Keywords: Occupational mortality, Occupational accidents, Colombia.
Autor de correspondencia

* Bacterióloga. Docente Universidad de los Llanos. Correo: 1plata@unillanos.edu. co. Orcid: https://orcid.org/0000-0002-03758875. Villavicencio Meta. 
ISSN-PRINT

1794-9831

E-ISSN 2322-7028

Vol. 16 No. 2

May - Ago 2019

Cúcuta, Colombia

\section{Resumo}

Introdução: A mortalidade é considerada um indicador do estado da saúde da população, porém, é fundamental reconhecer o seu comportamento epidemiológico. Objetivo: Determinar o comportamento epidemiológico da mortalidade por acidentes de trabalho que aconteceram no estado do Meta, durante o período entre 2010 e 2015. Materiais e métodos: Estudo descritivo com análise do registro único de afiliações processada no SPSS. Foi empregada a estatística descritiva, taxas de mortalidade e a análise de tendências. Resultados: A tendência dos casos de mortalidade por acidentes de trabalho tem diminuído, o $81 \%$ da variabilidade dos casos de mortalidade explica-se pela sua relação com os anos. Conclusão: Os projetos, programas e politicas de saúde devem mostrar um acompanhamento e monitoramento aos programas de promoção da saúde do trabalhador. Entretanto, deve-se avaliar o impacto dessas ações assim como ser priorizados e realizados ajustes.

Palavras-chave: Mortalidade ocupacional, acidentes de trabalho, Colômbia

\section{Introducción}

El proceso de medición y vigilancia en salud pública registra el número de casos de mortalidad según codificación internacional de enfermedades CIE 10, estos casos son susceptibles de ser utilizados para identificar las tendencias epidemiológicas.

La dinámica de los análisis de las tendencias de mortalidad en las poblaciones, permite identificar el conjunto de situaciones objetivas o características, que conforman aquello que se denomina 'la carga de enfermedad' que padece un grupo social en particular o la población en general. Desde la perspectiva del desarrollo humano, hay que visibilizar las condiciones sanitarias que definen a una población para documentar las tendencias, los logros obtenidos, la agenda pendiente y los nuevos desafíos en el abordaje del tema de su salud. Para las enfermedades en cuya causa o evolución intervienen las condiciones de trabajo, es decir, las enfermedades laborales, el análisis es limitado, lo que constituye uno de los principales obstáculos para el desarrollo de las necesarias estrategias $\mathrm{y}$ acciones preventivas.

Algunos autores plantean que los accidentes de trabajo constituyen una cuantiosa fuente de generación de costos $(1-4)$, los cuales aumentan con rapidez como lo indica un informe de la Organización Internacional del Trabajo (OIT) (5). Aunque fijar un valor a la vida humana es imposible, las cifras de indemnización demuestran que el costo de las enfermedades representa cerca del $4 \%$ del producto interno bruto mundial. Estos gastos se asocian a ausentismo laboral, tratamientos de enfermedades, incapacidad y prestaciones de supervivientes $(2,5-7)$.

A nivel internacional y nacional se observan cifras alarmantes de ocurrencia de accidentes laborales. Los cálculos más recientes de la OIT, revelan que hay 2 millones de fallecimientos anuales y 270 millones de accidentes relacionados con el trabajo, más de 5000 al día, y por cada accidente mortal hay entre 500 y 2 000 lesiones, según el tipo de trabajo (8). Estadísticas como estas, muestran la necesidad de realizar investigaciones científicas que contribuyan a disminuir estos indicadores. La mejora de las condiciones laborales evita la ocurrencia de estos hechos, y un bienestar físico, psíquico y social del factor humano que realiza sus funciones en los ambientes de trabajo $(2,5)$.

Aunque la ausencia de datos analizados es generalizada a escala internacional, en la literatura existen algunos estudios que calculan de forma rigurosa la mortalidad por enfermedades laborales para poblaciones definidas de trabajadores. Formando parte de un informe sobre la carga global de enfermedad en el mundo (6) se calculó la incidencia de lesiones (accidentes traumáticos) y enfermedades de origen laboral mediante dos estrategias: el método directo, basado en la recopilación de la información disponible en los registros sanitarios en cada país, y el método indirecto, basado en la aplicación de tasas específicas de incidencia y mortalidad, a las correspondientes categorías de edad y sexo en la población trabajadora. Estos autores calculan que cada año ocurren en el mundo 100 millones de accidentes, 11 millones de casos nuevos de enfermedades laborales y 800.000 muertes (100.000 por accidentes de trabajo y el resto debidas a enfermedades) como consecuencia de exposiciones laborales.

Al revisar indicadores de la situación colombiana, se observa que desafortunadamente no se detienen ni disminuyen los accidentes mortales; las cifras de accidentes, enfermedad y muerte laboral han aumenta- 
do en todos los casos, y las de cobertura siguen siendo insuficientes, no cubren la totalidad de la población trabajadora del país (7).

Como Colombia no cuenta con un sistema de información oficial por parte del Ministerio de Trabajo, fue necesario acudir a los reportes de Fasecolda (7), los cuales indican que en 2014, pese a que hubo mejoría en los índices de empleo y cobertura, la exclusión continuó, solo se presentaron 455.130 puestos de trabajo más, con un pequeño repunte en la cobertura en riesgos laborales (se afiliaron 665.797 personas más) recuperándose así la disminución que la cobertura tuvo en 2013. Por otra parte, la cobertura promedio anual en 2014 representó un 42\% de la población empleada, lo que deja por fuera de la protección al $58 \%$, o sea a más de 12'568.000 de trabajadores; sin embargo, en el primer semestre de 2015 hubo 530.000 nuevas afiliaciones, lo que aumentó un punto la cobertura.

La mortalidad en el trabajo según su causa, puede ser por enfermedad laboral (EL) o por accidente de trabajo (AT) (9). Generalmente las muertes se presentan por causa de accidentes de trabajo, y son muy pocos los casos reconocidos por enfermedad laboral. De acuerdo a la evolución, del total de muertes calificadas entre 2010 y 2014, el promedio anual fue de 670 muertes en el trabajo, solo dos de ellas a causa de una enfermedad calificada como laboral (7). La OIT estima que de las cerca de 2,3 millones de muertes al año relacionadas con el trabajo, más de 350.000 son causadas por accidentes y las restantes son provocadas por enfermedades laborales (8).

El Plan Decenal de Salud Pública 2012 - 2021, en su dimensión de salud y ámbito laboral propone políticas e intervenciones sectoriales y transectoriales que buscan el bienestar, la protección de la salud y el fomento de las intervenciones que modifican positivamente las situaciones y condiciones para la salud de los trabajadores del sector formal e informal de la economía, a través de estrategias enmarcadas en los siguientes componentes: la seguridad y salud en el trabajo y la atención de situaciones prevalentes de origen laboral (10).

La ampliación del conocimiento sobre accidentalidad laboral es importante; los costos de una mortalidad laboral son altos para las familias, empresas y sociedad e incluyen costos humanos y económicos que abarcan aspectos como el dolor y sufrimiento, afectación del equipo de trabajo, productividad empresarial y gastos sanitarios, entre otros. Es importante identificar la magnitud de los problemas de salud integral, cómo han evolucionado en años recientes, cuáles son los grupos en riesgo y cuál es su entorno socioeconómico, cultural y territorial.

Esta investigación permitirá reconocer el comportamiento de la mortalidad por accidente laboral en el departamento del Meta, identificar qué grupos poblacionales son los más afectados e inferir sobre los determinantes poblacionales que degradan la salud de los habitantes en los municipios de este departamento. Igualmente, aportará conocimientos al desarrollo científico debido a que los resultados serán referente regional en la descripción de las tendencias de mortalidad, los determinantes poblaciones y el comportamiento del departamento. También se ofrecerán resultados que se podrán aplicar en la planificación del desarrollo regional y en la implementación de programas de salud pública que sirvan a la protección de la vida y el bienestar de la población, a la disminución de la mortalidad y a la pérdida potencial de años de vida. A nivel nacional contribuirá al desarrollo de la vigilancia epidemiológica y la salud pública.

Del mismo modo, este estudio se constituirá en un aporte al desarrollo nacional, contribuyendo al reconocimiento del análisis de información periódica como factor de gran influencia para la planeación y toma de decisiones por parte de los líderes políticos. A partir del análisis se pueden desarrollar investigaciones que generen estrategias de protección de la salud del trabajador, que aporten positivamente a la problemática del contexto regional y que sirvan de apertura al campo del cuidado de la salud y la prevención en el contexto laboral.

\section{Objetivos}

\section{Objetivo General}

Determinar la epidemiología de los casos de mortalidad por accidente laboral ocurridos en el departamento del Meta, 2010-2015.

\section{Objetivo específico}

Identificar las características sociodemográficas de la población fallecida por esta causa, estimar la magnitud mediante tasas de mortalidad y describir la tendencia en la línea del tiempo de los casos. 
ISSN-PRINT

1794-9831

E-ISSN 2322-7028

Vol. 16 No. 2

May - Ago 2019

Cúcuta, Colombia

\section{Materiales y Métodos}

Para cumplir con los objetivos, el estudio se realizó en cuatro fases. La primera incluyó la validación estadística y epidemiológica, para revisión de categorías de variables, clasificación de variables, verificación de casos confirmados y ajustados, verificación del lugar de procedencia y la introducción de etiquetas en códigos, valores y perdidos. En la fase dos se determinó la tasa de mortalidad para cada municipio; la fase tres permitió el análisis de las tendencias utilizando los indicadores simples de la fase dos; y finalmente se identificaron las características sociodemográficas, mediante estadística descriptiva, en la fase cuatro.

Se realizó un estudio epidemiológico observacional, descriptivo y retrospectivo en el Departamento del Meta, entre los años 2010 a 2015. Se utilizaron las bases de datos de uso nacional del sistema de información del registro único de afiliaciones (RUAF) años 2010 - 2015; las cuales contienen los registros de los eventos procedentes de las atenciones médicas realizadas en los diferentes centros de salud, clínicas y hospitales de la red de Instituciones prestadoras de salud públicas y privadas del departamento. En este sentido, cada uno de los eventos fueron consignados en certificados de defunción diligenciados por el personal médico, mediante entrevistas estructuradas y estandarizadas a familiares y la revisión de la historia clínica, que contienen las variables de persona, tiempo y lugar.

La base de datos RUAF (2010 - 2015) contenía 23647 registros, la validación estadística y epidemiológica y la aplicación del criterio de inclusión, lugar de procedencia y/o residencia en el departamento del Meta; datos que permitieron la toma de 217 unidades de análisis, bajo criterios epidemiológicos descritos para los eventos y en los lineamientos nacionales, que luego fue sometida al análisis de caracterización por la investigadora.

Las variables de análisis fueron: sexo, edad, municipio de procedencia, área de residencia, lugar de defunción, año del reporte, nivel educativo, aseguramiento y causa de muerte.
A continuación se describieron las variables a través del uso de la estadística descriptiva (para las variables cualitativas se emplearon frecuencias y porcentajes, y para las cuantitativas medidas de tendencia central y variabilidad), utilizando para ello el software SPSS versión 23 licenciado, propiedad de la Universidad de los Llanos.

La construcción de las tasas por municipio se realizó usando como denominador la población municipal para el período, según reporte del Departamento Nacional de Estadística (DANE). Todas las tasas fueron calculadas empleando una constante de 100.000 habitantes.

Este estudió contó con el respectivo aval ético del Comité de la Universidad de los Llanos y mantuvo todos los requisitos exigidos por la Resolución 8430 de 1993 para la investigación en salud en Colombia (11). No representa riesgo real ni potencial sobre los sujetos, ya que el método de recolección es documental, razón por la cual, no se realizó ninguna intervención o modificación intencionada en los sujetos de estudio. No se usaron nombres o números de documentos de identidad, con el fin de no comprometer la confidencialidad.

\section{Resultados}

Para una mejor comprensión de los resultados, estos se presentarán según las fases propuestas.

Fase uno: la validación estadística y epidemiológica permitió conocer que el 0,92\% (217) de los registros correspondieron a mortalidad por accidentes laborales.

Fase dos: respecto a la tasa de mortalidad para cada municipio, se encontró que los que presentaron las mayores tasas de mortalidad por cada 100000 habitantes fueron: en 2010 Barrranca de Upia y Cabuyaro; en el 2011 Cubarral y Puerto Gaitán; en el año 2012 El Dorado y Guamal; en 2013 La Uribe y Macarena; en 2014 Mesetas y la Uribe; y, en 2015 Mesetas y Puerto López (Tabla 1). 
Tabla 1. Tasa de Mortalidad por accidente laboral por año, por municipio, Departamento del Meta, años 2010 - 2015

\begin{tabular}{|c|c|c|c|c|c|c|}
\hline \multirow{2}{*}{ Municipios del Meta } & \multicolumn{6}{|c|}{ AÑO } \\
\hline & 2010 & 2011 & 2012 & 2013 & 2014 & 2015* \\
\hline Villavicencio & 7,9 & 3,6 & 4,6 & 3,9 & 3,8 & 1,4 \\
\hline Acacías & 0,0 & 4,8 & 4,7 & 4,6 & 0,0 & 0,0 \\
\hline Barranca de Upía & 28,2 & 0,0 & 0,0 & 0,0 & 0,0 & 0,0 \\
\hline Cabuyaro & 26,1 & 0,0 & 0,0 & 0,0 & 0,0 & 0,0 \\
\hline Castilla la Nueva & 0,0 & 0,0 & 11,4 & 0,0 & 0,0 & 0,0 \\
\hline Cubarral & 0,0 & 35,5 & 0,0 & 0,0 & 0,0 & 0,0 \\
\hline Cumaral & 11,5 & 0,0 & 11,3 & 0,0 & 0,0 & 0,0 \\
\hline El Calvario & 0,0 & 0,0 & 0,0 & 0,0 & 0,0 & 0,0 \\
\hline EI Castillo & 0,0 & 0,0 & 0,0 & 15,5 & 15,6 & 0,0 \\
\hline El Dorado & 0,0 & 0,0 & 29,4 & 0,0 & 0,0 & 0,0 \\
\hline Fuente de Oro & 0,0 & 0,0 & 0,0 & 0,0 & 0,0 & 0,0 \\
\hline Granada & 3,6 & 1,7 & 0,0 & 1,7 & 1,6 & 0,0 \\
\hline Guamal & 10,9 & 10,9 & 21,6 & 0,0 & 0,0 & 0,0 \\
\hline Mapiripán & 6,6 & 12,8 & 12,5 & 6,1 & 5,9 & 0,0 \\
\hline Mesetas & 0,0 & 18,1 & 0,0 & 0,0 & 26,7 & 8,9 \\
\hline La Macarena & 7,1 & 0,0 & 3,3 & 19,3 & 3,1 & 0,0 \\
\hline Uribe & 7,0 & 0,0 & 0,0 & 19,5 & 19,0 & 0,0 \\
\hline Lejanías & 0,0 & 0,0 & 0,0 & 10,6 & 0,0 & 0,0 \\
\hline Puerto Concordia & 5,5 & 5,3 & 0,0 & 10,1 & 0,0 & 0,0 \\
\hline Puerto Gaitán & 5,6 & 22,1 & 0,0 & 10,9 & 0,0 & 5,4 \\
\hline Puerto López & 3,2 & 3,2 & 15,6 & 9,2 & 3,0 & 6,0 \\
\hline Puerto Lleras & 0,0 & 19,6 & 0,0 & 0,0 & 0,0 & 0,0 \\
\hline Puerto Rico & 5,5 & 5,5 & 0,0 & 0,0 & 0,0 & 0,0 \\
\hline Restrepo & 0,0 & 0,0 & 9,5 & 0,0 & 0,0 & 0,0 \\
\hline San Carlos de Guaroa & 0,0 & 12,1 & 0,0 & 0,0 & 0,0 & 0,0 \\
\hline San Juan de Arama & 0,0 & 0,0 & 0,0 & 11,2 & 0,0 & 0,0 \\
\hline San Juanito & 0,0 & 0,0 & 0,0 & 0,0 & 0,0 & 0,0 \\
\hline San Martín & 0,0 & 4,3 & 0,0 & 0,0 & 0,0 & 0,0 \\
\hline Vistahermosa & 4,3 & 12,7 & 4,1 & 4,1 & 8,0 & 3,9 \\
\hline TOTAL & 5,7 & 4,6 & 4,4 & 4,6 & 3,3 & 1,2 \\
\hline
\end{tabular}

* Para el año 2015 las bases no se encuentran con todos los registros.

Fuente: Base de datos de mortalidad, años 2010 a 2015.

Fase tres: el análisis de la tendencia de los casos de mortalidad por accidentes laborales tiende a la disminución, el $81 \%$ de la variabilidad de los casos de mortalidad, es explicada por su relación con los años (gráfico 1). 
ISSN-PRINT

1794-9831

E-ISSN 2322-7028

Vol. 16 No. 2

May - Ago 2019

Cúcuta, Colombia

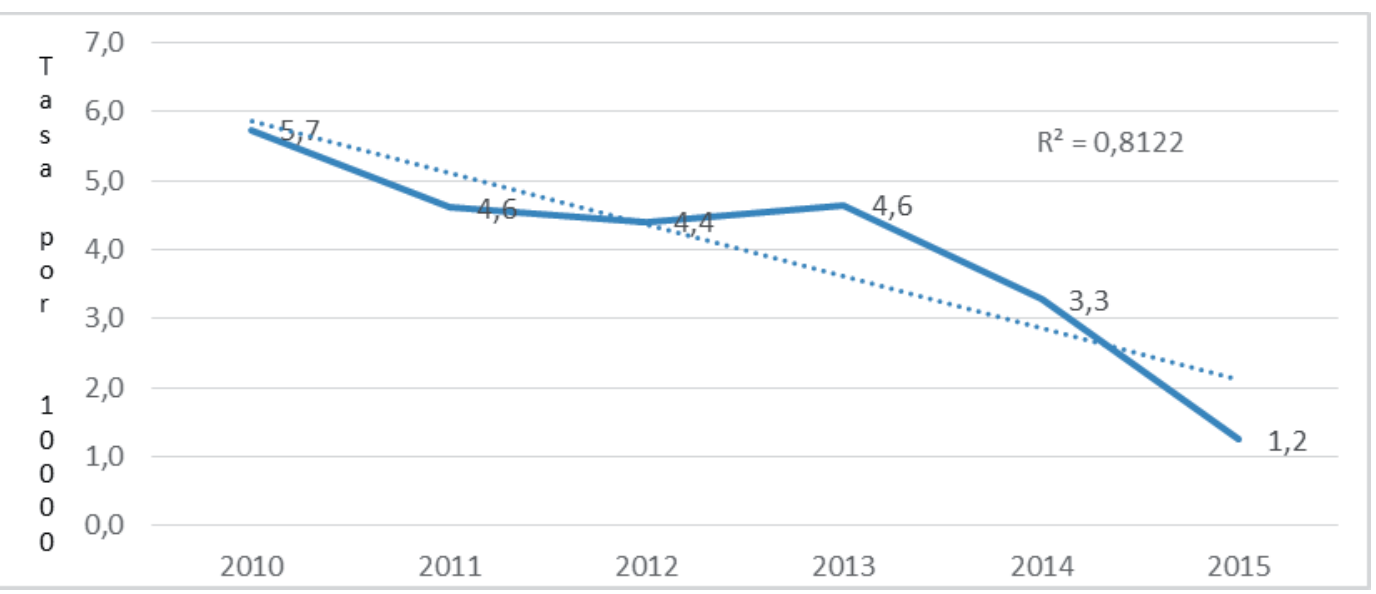

Gráfico 1. Casos de mortalidad por accidentes laborales (2010-2015).

Fuente: autora.

Fase cuatro: la identificación de las características sociodemográficas estableció que el 95,4\% de los fallecidos pertenecía al sexo masculino. El $50 \%$ de los muertos por accidentes laborales en el Meta durante el período de estudio tenía 33 años o menos; siendo 25 años la edad que más se repite. Los municipios que más reportaron muertes fueron Villavicencio, Puerto López, Macarena y Acacias. El 63.3\% de los fallecidos procedían de la cabecera municipal, el 4,6\% del área rural dispersa y el $18,9 \%$ del centro poblado. El 30,4\% de las defunciones ocurrió en instituciones prestadoras de servicios de salud, el $28,1 \%$ vía pública y el $16,6 \%$ en el lugar de trabajo.

Los años que reportaron mayor cantidad de defunciones fueron 2010 y 2013. El año con menor aporte fue el 2014 con 14,3\% de las muertes. El 45,7\% tenía un estado conyugal de convivencia con pareja o casado, al momento de la mortalidad; el 32,2\% estaba soltero; el $8,3 \%$ separado o divorciado; y del $20,7 \%$ no se obtuvo información.

Del 58\% de los fallecidos, no se obtuvo información acerca del último año de estudios aprobado. Entre quienes tuvieron algún grado educativo aprobado se encontró que el 15,7\% tuvo básica primaria, el 5,3\% básica secundaria, el 13,8\% media académica, el $4,6 \%$ profesional. El mayor grado referenciado como último año de estudios aprobado fue maestría con el 1,3\%. El 13,3\% pertenecía al régimen subsidiado, el $29 \%$ al contributivo, el $22,6 \%$ no estaba asegurado. Del $6,4 \%$ no se obtuvo información.

Respecto a la variable causa de muerte, las tres principales fueron: agresiones con disparos o explosivos
$(32,2 \%)$, accidentes en vehículo (12\%); otras causas $(30,4 \%)$, exposición a electricidad $(7,8 \%)$, golpes o caídas $(5,5 \%)$, fenómenos naturales $(3,2 \%)$ y ahogamiento $(2,8 \%)$

\section{Discusión}

Este estudio aporta evidencias para seguir construyendo en el departamento del Meta y la región de Orinoquía, el panorama de la mortalidad por accidente laboral, siendo uno de los indicadores mínimos de Seguridad y Salud en el Trabajo (SST) determinados por el artículo 15 de la Resolución 1111 de 2017 (12). En este estudio la tasa de mortalidad general disminuyó a través de los años y la población económicamente activa fue mayormente afectada. Igualmente, se encuentran diferencias entre municipios, algunos sin reporte de casos y con tendencia a la disminución de AT y EL. Por tanto, será necesario contemplar elementos claves que midan el impacto de las actividades de promoción y prevención que se realizan en las empresas y las Administradoras de Riesgos Laborales (ARL), siguiendo los lineamientos de políticas emanadas del Gobierno Nacional a través del Ministerio de Salud y Protección Social y del Ministerio de Trabajo. En este sentido, se debe tener en cuenta que en los últimos años, la SST ha ido cobrando relevancia y posicionándose como un tema prioritario, debido a las alarmantes cifras de enfermedad y lesiones ocasionadas por el trabajo, que han ido aumentando gradualmente (13).

Por otra parte, los Objetivos de Desarrollo del Milenio (ODM) y los Objetivos de Desarrollo Sostenible (ODS) (14) a nivel global, definen un conjunto de acciones que tienen una importante influencia en la me- 
jora de las condiciones de vida de las personas en el mundo. El logro de dichos objetivos está relacionado íntimamente con los avances en SST. En particular, objetivos como garantizar una vida sana y promover el bienestar para todos en todas las edades está muy relacionado con la salud en el trabajo para las personas en edad productiva, así como su formalización, a fin de garantizar la cobertura en seguridad social de las familias y el incremento del bienestar general.

No obstante, de acuerdo con el análisis de accidentalidad laboral en Colombia, la mortalidad por accidentes laborales es la segunda causa importante de muerte, con una tendencia al descenso a través del tiempo, siendo mayor, para el sexo masculino (1516); situación similar a la encontrada en este estudio, donde la proporción hombre: mujer fue de 6:1. Sin embargo, difiere de países como España (17) y Brasil (18), donde las más afectadas son las mujeres, al ser discriminadas en el mercado laboral y sujetas a condiciones precarias, con menor sueldo y menos oportunidades de ascenso en los trabajos.

La diferencia por sexo puede tener explicación debido a que los hombres se emplean más en aquellos sectores con altas incidencias de accidentalidad, algunos autores mencionan que se debe a las tareas desempeñadas $(19-23)$, sobretodo porque muchos más hombres que mujeres laboran en ocupaciones que los exponen a accidentes; de ahí que sean más altas las probabilidades que sufran accidentes fatales o de otro tipo por causas relacionadas con el trabajo. Es decir, que los hombres tienen más presencia en industrias como la construcción y la minería, donde se presentan más casos de moralidad laboral; mientras que la gran mayoría de las mujeres trabajadoras se concentran en la agricultura y el sector de servicios. Desde otra perspectiva, los investigadores también demostraron que los hombres tienden a adoptar menos medidas preventivas y de protección para efectuar su trabajo respecto de las mujeres, razón por la cual en el sexo masculino predomina más la mortalidad de accidentes laborales.

Si se tiene en cuanta la edad, se puede establecer que es similar a lo encontrado en diferentes investigaciones en Colombia (19-23) y en otros países (21-22). En este sentido, son los jóvenes quienes deben sobrevivir, y por ese motivo son los primeros en llenar las filas del desempleo; al mismo tiempo, son potencialmente explotables por el capital, vistos como consu- midores, clientes y operarios, puesto que tienen toda la energía, las ideas y el potencial.

De igual forma, se debe tener en cuenta que la falta de información sobre calidad y de consciencia por parte de los involucrados, contribuye a que se presenten dos problemas importantes: por un lado, se encuentra la insuficiente colaboración entre los entes gubernamentales de tipo laboral y de salud, para organizar y garantizar el adecuado funcionamiento de los servicios de salud preventivos y curativos para la población trabajadora, así como el establecimiento de una relación fuerte y sostenida con la atención primaria; y por el otro, la cobertura de los servicios de SST sigue siendo muy baja. Esto supone que no existen y que a menudo no se cumple con las expectativas de los trabajadores, sobre todo por el bajo acceso a las intervenciones preventivas básicas y a las medidas de protección contra las enfermedades profesionales, hecho que afecta su capacidad de trabajo (24), evidenciado así una cobertura baja de afiliación al régimen contributivo de quienes fallecieron en el departamento del Meta.

Es de hacer notar, que la baja cobertura puede encontrar explicación en el hecho de que las organizaciones prestadoras de servicios de SST tienen su interés centrado en la dinámica del mercado, buscando esencialmente la rentabilidad, hecho que no garantiza la calidad de los mismos (25).

De otra parte, se debe precisar que la población más afectada suele ser la más vulnerable, entiéndase trabajadores del sector informal, agropecuario (26-28). Por año de ocurrencia, el año que reportó menor tasa de accidentes laborales fue 2015, debido particularmente a la falta de información consolidada; sin embargo, se observa una tendencia a la disminución a través de los años analizados. En Inglaterra y Gales la reducción se atribuye a prácticas de trabajo más seguras, reducción en el número de hombres empleados en trabajos más peligrosos y la disminución de la mortalidad por algunas enfermedades en toda la población (29).

De modo similar, se debe señalar que la información sobre las causas de muerte permite determinar la magnitud de los problemas de salud de una población, definir políticas y programas en el sector de la salud, así como realizar el seguimiento y la evaluación del impacto que producen las enfermedades sobre una determinada población (30-31). Las agresiones con disparos y los accidentes en vehículos son las causas 
ISSN-PRINT

1794-9831

E-ISSN 2322-7028

Vol. 16 No. 2

May - Ago 2019

Cúcuta, Colombia más relevantes de mortalidad en el Meta, lo cual es diferente a lo reportado en la literatura (23-26), relacionado quizá con las características poblacionales, culturales y territoriales particulares de la región.

Del mismo modo, otros informes nacionales (32-33) concuerdan al indicar que los departamentos donde hay más mortalidad por accidente laboral son Cundinamarca, Antioquia, Meta y Valle del Cauca, siendo la principal razón la necesidad de sustento económico, lo que obliga a aceptar salarios mínimos y sobrecarga laboral.

Es por este motivo, que la legislación colombiana le ha dado paso a la implementación del Sistema General de Seguridad y Salud en el Trabajo en las empresas del sector privado y público (34), lo cual es muy conveniente y se espera incida en la reducción de la mortalidad por AT y EL.

Por tanto, se debe resaltar que los datos aquí obtenidos son un importante aporte a las autoridades como el Ministerio de Salud y Protección Social, el Ministerio del Trabajo, los Comités Nacional y Seccionales de Seguridad y Salud en el Trabajo, quienes realizan los planes Nacional y seccionales de Seguridad y Salud en el Trabajo; toda vez que se hace necesario un vínculo entre la ciencia y la política, que posibilite el desarrollo de políticas públicas basadas en la evidencia científica (35).

También se debe precisar, que la falta de consolidación de los datos existentes, especialmente para el año 2015, impone un límite al análisis de la información, lo cual deberá mejorarse a futuro. Es por eso, que se deben obtener fuentes de información con datos que permitan unos análisis más detallados y por tanto con resultados más sólidos que apoyen la evidencia de la problemática sanitaria de los metenses. Esta investigación presentó limitaciones derivadas de la utilización de fuentes secundarias que fueron solucionadas con la validación de la información.

Por último, se debe precisar que para futuros estudios será necesario profundizar en las situaciones culturales, regionales y de contexto que van más allá del enfoque analítico y del diseño propuesto, y así profundizar en la explicación de los resultados.

\section{Conclusiones}

- El perfil del fallecido por accidentes laborales en el departamento del Meta en el período de estudio fue de un hombre de 33 años o menos, con bajo nivel de escolaridad, no afiliado al sistema General de Seguridad Social y procedente del área urbana.

- Las diferencias de las principales causas de muerte por accidentes laborales entre municipios, deben estar en relación con la priorización de intervenciones, el destino de los recursos y la necesidad de investigación aplicada.

- Los proyectos, programas y políticas de salud deben reflejar un seguimiento y monitoreo a los programas de promoción de la salud laboral; igualmente, se debe tener en cuenta la evaluación del impacto y la realización de ajustes a la priorización por parte de las autoridades competentes.

\section{Conflicto de Intereses}

La autora declara no tener ningún conflicto de interés

\section{Referencias Bibliográficas}

1. Ashby S, Diacon S. Motives for occupational risk management in large UK companies. Safety Science, 1996; 22(1):229-243.

2. Beramendi C. Impacto económico de los accidentes de trabajo y sus factores asociados en un hospital de cuarto nivel de Essalud. Universidad Nacional Mayor de San Marcos. 2004.

3. Forastieri V. El tiempo perdido por accidentes laborales. Revista Seguridad y Medio Ambiente [Internet], 2009 [consultado el 20 mayo de 2017]; 115:6-15. Disponible en: http://www.mapfre.com/ fundacion/html/revistas/seguridad/n115/entrevista.html

4. Saari J. La prevención de accidentes hoy en día. Revista de la Agencia Europea para la Seguridad y la Salud en el Trabajo. 2002; 4:3-5.

5. Milan Jorge IE. Ergonomía participativa para la prevención de accidentes industriales. [Tesis en Internet]; Universidad Nacional Autónoma De México; 2013 [consultado el 16 agosto de 2017]. Disponible en: http://132.248.52.100:8080/xmlui/handle/132.248.52.100/2117 
6. Organización Panamericana de la Salud. Mejoramiento de la estructura y desempeño de los sistemas nacionales de información en salud. Enfoque operacional y recomendaciones estratégicas. Santiago de Chile: Oficina de Representación OPS/ OMS. 2009.

7. Federación de aseguradoras colombiana (Fasecolda). Cámara técnica de riesgos laborales. [Internet]. Colombia; 2015 [consultado 26 de marzo de 2017]; p. 24-65. Disponible en: http://www.fasecolda.com/files/6014/4975/9062/Presentacin - Simposio Internacional ORP - Dr. Botero.pdf

8. Organización Inernacional del Trabajo. Tendencias mundiales sobre accidentes del trabajo y enfermedades profesionales. Informe elaborado para el día mundial de la seguridad y la salud en el trabajo, 28 de abril de 2015. [Internet]. Colombia; 2015 [consultado el 23 de marzo de 2017] Disponible en: http:// www.ilo.org/legacy/english/osh/es/story_content/external_files/fs_st_1-ILO 5 es.pdf.

9. Congreso de la República de Colombia. "Por La Cual Se Modifica El Sistema De Riesgos Laborales Y Se Dictan Otras Disposiciones En Materia De Salud Ocupacional. Bogotá D.C.: Senado de la República de Colombia; 2012 [consultado 11 de junio de 2017.Disponible en: https://www.minsalud.gov.co/ sites/rid/Lists/BibliotecaDigital/RIDE/DE/DIJ/Ley-1562-de-2012.pdf

10. Congreso de la República de Colombia. Plan decenal de salud pública 2012 - 2021. [Internet]. Bogotá D.C.: Senado de la República de Colombia; 2013 [consultado el 11 de junio de 2017]. Disponible en: https://www.minsalud.gov.co/Documentos\%20y\%20Publicaciones/Plan\%20Decenal\%20 -\%20Documento\%20en\%20consulta\%20para\%20aprobaci\%C3\%B3n.pdf.

11. República de Colombia. Ministerio de Salud. Resolución No 008430 de 1993, octubre 4, por la cual se establecen las normas científicas, técnicas y administrativas para la investigación en salud. Santa Fe de Bogotá: El Ministerio; 1993.

12. Alcaldía Mayor de Bogotá. Resolución 1111/2017 de marzo 27. Diario oficial, No 50189. Por la cual se definen los Estándares Mínimos del Sistema de Gestión de Seguridad y Salud en el Trabajo para empleadores y contratantes. Santa Fe de Bogotá, 2017.

13. Cordia C. De una promoción de la salud en el lugar de trabajo hacia la gestión integradora de la salud en el lugar de trabajo: Tendencias y evolución. Red mundial de salud ocupacional. 2003; $6: 1-4$.

14. Organización internacional del Trabajo. Plan de implementación de la OIT Agenda 2030 para el Desarrollo Sostenible. Disponible en: https://www.ilo.org/wcmsp5/groups/public/---dgreports/---dcomm/--webdev/documents/publication/wcms 510124.pdf

15. Ministerio de Trabajo. Indicadores del sistema general de Riesgos Laborales cifras 2011-2015. [Internet]. Colombia; 2015 [consultado el 16 de marzo de 2017] Disponible en: Disponible en: http:// fondoriesgoslaborales.gov.co/documents/infoestadistica/2015/INFORME\%20INDICADORES\%20 SGRL $\% 202011 \% 20-\% 202015 . p d f$

16. Harris C, Palmer K, Cox V, Darnton A, Osman J, Coggon D. Trends in mortality from occupational hazards among men in England and Wales during 1979-2010. BMJ Journals. 2016, 73: 385-393.

17. Garnica M. Caracterización de los accidentes de trabajo biológicos del personal de servicios generales que laboro en instituciones hospitalarias del país. [Tesis magister en internet]. Bogotá, Colombia: Universidad Javeriana; 2011 [consultado el 4 de noviembre de 2016]. 145 p. Disponible en: https:// repository.javeriana.edu.co/handle/10554/13785.

18. Santana M, Monteiro M. Saúde do trabalhador \& terceirização: perfil de Trabalhadores de serviço de limpeza hospitalar. Rev. Latino-am Enfermagem. 2004, 12(2):17-23.

19. Pérez M, Muñoz A. Accidentalidad laboral reportada por las empresas afiliadas a una Administradora de Riesgos Laborales, enero - diciembre, 2013. Rev. Fac. Nac. Salud Pública 2014; 32(2): 67-75

20. Dirección General de Seguridad y Salud Laboral - Junta de Andalucía, Consejería de empleo. Estadísticas de accidente de trabajo y enfermedades profesionales en Andalucía. 2013-2014 [internet]. Andalucia, España; 2015 [consultado el 14 de noviembre de 2016]. Disponible en: http://www.juntadeandalucia.es/em- 
ISSN-PRINT

1794-9831

E-ISSN 2322-7028

Vol. 16 No. 2

May - Ago 2019

Cúcuta, Colombia

pleo/www/seguridad-y-salud-laboral/informacion-en-ssl/documentos/estadisticas_siniestros/2010 cuaderno enero junio.pdf

21. García C, Carbajosa M, Llopis C, Rico A, Jurado C, et al. Muertes traumáticas por accidentes laborales en Sevilla.2011-2014. Cuad. med. Forense [online]. 2008, n.52 [citado consultado 11 de marzo de 2017]; 52:137-146. Disponible en: http://scielo.isciii.es/scielo.php?script=sci_arttext\&pid=S113576062008000200004\&lng=es\&nrm=iso.

22. Muñoz J, Daponte A, López L, Mateo I. Influencia de las características individuales y de las condiciones laborales en la gravedad de las lesiones por accidente de trabajo registradas en Andalucía. Rev. Esp. Salud Pública. [Internet]. 2013 [consultado el 16 de abril de 2017]; 83(6):847-861. Disponible en: http:// scielo.isciii.es/scielo.php?script $=$ sci_arttext\&pid=S1135-57272009000600008\&lng $=$ es\&nrm=iso

23. Fondo de Riesgos Laborales. Mortalidad de los accidentes laborales en Colombia entre 2015 y 2016. [internet]. Bogotá, Colombia; 2016 [consultado el 16 de octubre de 2017]. Disponible en: https:// safetya.co/mortalidad-de-los-accidentes-laborales-colombia-2015-2016/.

24. Varona M, Torres C, Díaz S, Palma R, Checa D, Conde J. Estado de la oferta técnica de servicios de higiene y seguridad industrial, Colombia, 2010. Biomédica. 2012;32:60-70.

25. Tabares L, López A. Salud y riesgos ocupacionales por el manejo de plaguicidas en campesinos agricultores, municipio de Marinilla, Antioquia, 2009. Rev. Fac. Nac. Salud Pública 2011; 29(4): 432-444.

26. Bedoya E, Behaine B, Gómez E, Burgos Y, Carrillo M, et al. Control de pérdidas por accidentalidad laboral en el sector logístico. Espacios. [internet]. 2018 [consultado el 16 de octubre de 2017]. 39(09):6. Disponible en: http://www.revistaespacios.com/a18v39n09/a18v39n09p06.pdf.

27. Vargas B, Quiroz P. Alteraciones neuropsicológicas en escolares de un municipio con niveles elevados de vapor de mercurio medioambiental, Colombia, 2008-2009. Rev. Fac. Nac. Salud Pública 2011; 29(4): 461-468.

28. Katikireddi S, Leyland A, McKee M, Ralston K, Stuckler D. Occupational mortality rates in the UK: Geographical comparisons using linked administrative data: Srinivasa Katikireddi. European Journal of Public Health. 2016, 26(1):217-223.

29. Holzman DC. Mountaintop Removal Mining. Digging into community health concerns. Environmental Health Perspectives. 2011;119(11):476-84.

30. Cordy P, Veiga M, Crawford B, Garcia O, Gonzalez V, Moraga D, et al. Characterization, mapping, andmitigation of mercury vapour emissions from artisanal mining gold shops. Environmental Research. 2012; 125:82-91.

31. Chakraborti D, Rahman M, Murrill M, Das R, Siddayya, Patil S, et al. Environmental arsenic contamination and its health effects in a historic gold mining area of the Mangalur greenstone belt of Northeastern Karnataka, India. J Hazard Mater. 2013; 15(262):1048-55.

32. Piedrahita H. La muerte en el trabajo. Fac.Nac. Salud Pública. 2002; 20(1):185-194

33. Federación de aseguradoras colombiana (Fasecolda). Cámara técnica de riesgos laborales. [Internet]. Colombia; 2015 [consultado el 14 de septiembre de 2017]; p. 24-65. Disponible en: http://www. fasecolda.com/index.php/ramos/riesgos-laborales/estadisticas-del-ramo/

34. Torres Satizábal L, Sanabria, M, Gómez G. El impacto de la legislación en riesgos laborales en los índices de accidentalidad, enfermedad y mortalidad de origen laboral en Colombia y Argentina. un caso de estudio. Perspectivas. 2018; 10: 66-74.

35. Monjardino T, Lucas R, Benavides F. Trabalho e Saúde em Portugal 2016, un primer informe sobre la salud laboral en Portugal. Arch Prev Riesgos Labor [Internet]. 2017 [citado 2019 Ene 20]; 20(1):6-8. Disponible en: http://dx.doi.org/10.12961/aprl.2017.20.01.1 